\title{
The relationship between traits optimism and anxiety and health-related quality of life in patients hospitalized for chronic diseases: data from the SATISQOL study
}

Sabrina Kepka ${ }^{1,2^{*}}$, Cédric Baumann ${ }^{3,4,5}$, Amélie Anota $^{6}$, Gaelle Buron $^{6}$, Elisabeth Spitz ${ }^{7}$ Pascal Auquier ${ }^{8}$, Francis Guillemin ${ }^{3,4,5}$ and Mariette Mercier ${ }^{1,6}$

\begin{abstract}
Background: The impact of psychological factors is often taken into account in the evaluation of quality of life. However, the effect of optimism and trait anxiety remains controversial and they are rarely studied simultaneously. We aimed to study the effect of this factor on health-related quality of life (HRQOL) of patients after a hospitalization in relation with their chronic disease.

Methods: Using cross-sectional data from the SATISQOL cohort, we conducted a multicentric study, including patients hospitalized for an intervention in connection with their chronic disease. Six months after hospitalization, patients completed a generic HRQOL questionnaire (SF-36), and the STAI and LOT-R questionnaires to evaluate optimism and trait anxiety. We studied the effect of each trait on HRQOL separately, and simultaneously, taking account of their interaction in 3 models, using an ANOVA.
\end{abstract}

Results: In this study, 1529 patients were included in three participating hospitals and there existed wide diversity in the chronic diseases in our population. The HRQOL score increased for all dimensions of SF36 between 15,8 and 44,5 when the level of anxiety decreased $(p<0.0001)$ for the model 1 , assessing the effect of anxiety on HRQOL and increased for all dimensions of SF36 between 3.1 and 12.7 with increasing level of optimism $(<0.0001)$ in the model 2 assessing the effect of optimism on HRQOL. In the model 3, assessing the effect of both anxiety and optimism on HRQOL, and their interaction, the HRQOL score for all dimensions of the SF36 increased when the level of anxiety decreased $(p<0.0001)$. It increased with increasing level of optimism $(p<0.006)$ in the model for all dimensions of SF36 except the Role Physical dimension. In this model, interaction between anxiety and optimism was significant for the Social Functioning dimension $(p=0.0021)$.

Conclusions: Optimism and trait anxiety appeared to be significantly correlated with HRQOL. Furthermore, an interaction existed between the trait anxiety and optimism for some dimensions of SF36. Contrary to optimism, it seems essential to evaluate trait anxiety in future studies about HRQOL, since it could represent a confounding factor.

Keywords: Quality of life, Chronic diseases, Validated questionnaires, Anxiety, Optimism

\footnotetext{
* Correspondence: sabrinakepka@yahoo.fr

'University of Franche-Comté, UPRES EA 3181, Besançon, France

${ }^{2}$ Emergency and Intensive Care Department $\mathrm{CHU}$, University Hospital Jean

Minjoz, 1 Bvd Fleming, Besançon 25030, France

Full list of author information is available at the end of the article
} 


\section{Background}

For the follow-up of chronic diseases, it is necessary to develop indicators that can easily be assessed, such as the measure of the health-related quality of life (HRQOL), which is governed by specific guidelines for implementation $[1,2]$. HRQOL as an indicator provides essential information to the clinicians to estimate the efficiency of their therapeutic and preventive actions [3,4]. Patients affected by chronic disease have a particular profile, due to their recourse to regular care and the necessity to adapt to their disease, and this can have consequences on HRQOL assessment. Thus, chronic diseases can generate psychological distress [5] and can be associated with a lower HRQOL [6]. The conceptual framework for our study is a variation on Broffenbrenner's ecological model [7], proposed by McLeroy [8], and explains the multiple levels of influence on health outcomes at both individual and environmental characteristics in HRQoL. The McLeroy model indicates five levels of influence: (a) intrapersonal factors (characteristics of individual such as personality traits, knowledge, attitudes, behavior, self-concept, skills, etc.), (b) interpersonal factors (formal and informal social support systems, including the family, work group, and friendship networks), (c) institutional factors (social institutions, organizations such as schools and healthcare facilities), (d) community factors (relationships among institutions and informal social networks in a defined area), and (e) public policy (local, state, and national laws and policies). For our proposed model, we considered only the influence at the individual level.

Some determinants of HRQOL such as gender, type of disease, age or socio-demographic characteristics (e.g. level of education, professional activity...) have been clearly identified in the literature. According to the original conceptual model of Wilson [9] reviewed by Ferrans [10], characteristics pertaining to both the individual and the environment can have an impact on the five major domains of HRQOL, namely biological and physiological factors, symptoms status, functional status, general health perceptions, and overall HRQOL. The effect of psychological characteristics has also been often evoked, but still warrants further exploration [11]. In this study we focus on the personal characteristics, particularly anxiety and optimism. Optimism and trait anxiety are characteristics inherent to every individual, and do not change over time or according to events with which the individual is confronted. Scheier and Carver theorized that the "disposition" towards optimism could be called "dispositional optimism" and proposed the notion of a measure for optimism $[12,13]$. They defined it as a relatively stable feature of the personality, which has important consequences on the way a person regulates their actions in the face of difficulties or stressful situations. For anxiety, Spielberger distinguished the notions of " state " and « trait » anxiety. He characterized trait anxiety as relatively stable individual differences in the tendency towards anxiety [14]. This tendency would be consistent according to different types of stressful situations and would be stable over time $[14,15]$.

Psychological factors could be considered as items determining the quality of life $[6,16]$. Furthermore, HRQOL and anxiety or optimism can influence how patients accept a diagnosis, and can be used as an outcome to evaluate the efficiency of a particular therapeutic approach $[17,18]$. However, the relation between these factors has never been specifically addressed, and available data in the literature do not yield a consensus regarding the role of these psychological factors in HRQOL.

Many studies have underlined the importance of optimism in the evaluation of HRQOL [4,19-23] but its impact remains controversial. Optimistic patients may have coping strategies characterized by better acceptance of the disease, and this can contribute to a lower risk of certain chronic diseases and as a result, better HRQOL [24-27]. Using negative coping has been reported to be associated with low levels of optimism and a high level of anxiety [28]. Anxiety is thus associated with a lower HRQOL [29-34]. Accordingly, pessimistic patients could have a lower HRQOL, exacerbated by anxiety or depression [21,35]. On the other hand, an association between optimism and anxiety or depression and HRQOL may no longer be significant after adjusting for anxiety, depression and sociodemographics variables [23]. Thus, the role of each of these factors has often been taken into account separately in the evaluation of HRQOL, and few studies have evaluated both simultaneously [23]. Furthermore, the results of studies published to date are divergent regarding the role of each factor. If these two factors are related to HRQOL, they are thus potential confounding factors, and it is therefore necessary to know the effect of each trait and to take it into account in the evaluation of HRQOL.

On the basis of Wilson's [9] and Ferrans models [10], the objective of this study is to clarify the relationships between trait anxiety, optimism and HRQOL. We hypothesized that the model has three causal pathways that contribute to the outcome variable, HRQOL: the anxiety-trait as a predictor $(\alpha)$, the impact of optimism as a moderator $(\beta)$, and the interaction of these two ( $\alpha$ and $\beta$ ) [36]. The moderator hypothesis is supported if the interaction is significant. There may also be significant main effects for the predictor and the moderator, but these are not directly relevant conceptually to testing the moderator hypothesis. We aimed to evaluate the relation between HRQOL and the traits optimism and anxiety among patients after hospitalization in relation to their chronic disease. 


\section{Methods}

\section{Design}

This study was performed using cross-sectional data from patients with chronic diseases included in the SATISQOL (SATisfaction and Quality Of Life) cohort study (Figure 1).

\section{Population and sample}

Patients were recruited during a stay in any of the 3 participating hospitals, in either medical or surgical departments. Patients were eligible if they were aged from 40 to 75 years old, hospitalized for the care of a chronic disease (defined as a disease ongoing for more than 6 months, and confirmed by a validation committee), and if the motive for hospitalization was an acute episode of the chronic disease, management of acute worsening of the disease, or initiation of new therapy for the chronic disease. We thus included any hospitalization for a medical or surgical intervention in connection with the chronic disease. Patients were excluded if they were hospitalized for a diagnostic assessment.

For the SATISQOL study, a HRQOL questionnaire was sent to each patient at 6 months and one year after discharge. Optimism and anxiety questionnaires were sent at 6 months only because they were considered to be stable over time. Clinical research assistants collected socio-demographic data and patient characteristics in each center. Subjects were informed about the study and written informed consent was obtained from all participants.

The SATISQOL study was approved by the national Institutional Review Board and the national committee for data protection (CCTIRS 07.212 and CNIL 1248560).
The SATISQOL cohort project was supported by an IRESP (Institut de recherche en santé publique) grant from Inserm, and a national hospital research grant from French Ministry of Health (PHRC, Programme Hospitalier de Recherche Clinique).

\section{Data collection}

The primary endpoint of our study was HRQOL at 6 months.

\section{Health-related quality of life (HRQOL)}

HRQOL was assessed using the validated French version of the MOS Short-Form 36 [37-39]. It includes 36 items, distributed in 8 dimensions: Physical Functioning (PF), Role Physical (RP), Bodily Pain (BP), Vitality, (VT) Social Functioning (SF), Role Emotional (RE), Mental Health $(\mathrm{MH})$ and General Health $(\mathrm{GH})$. A score was calculated for each dimension if more than half the items were completed. These scores were transformed to obtain a value between 0 (worst possible HRQOL) and 100 (best possible HRQOL) for each dimension.

\section{Anxiety}

Trait anxiety was estimated by the STAI (State Trait Anxiety Inventory) validated in French. Each answer to the 20 items of the questionnaire scored 1 to 4 . A global score ranging from 20 (the lowest level of anxiety) to 80 (the highest level of anxiety) was calculated if there were less than $20 \%$ missing data, corresponding to at least 17 items completed.

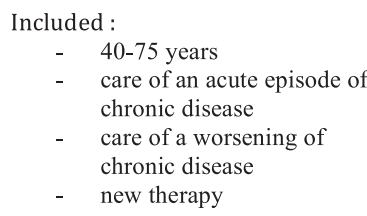

\section{SATISQOL Cohort} $\mathrm{n}=1657$

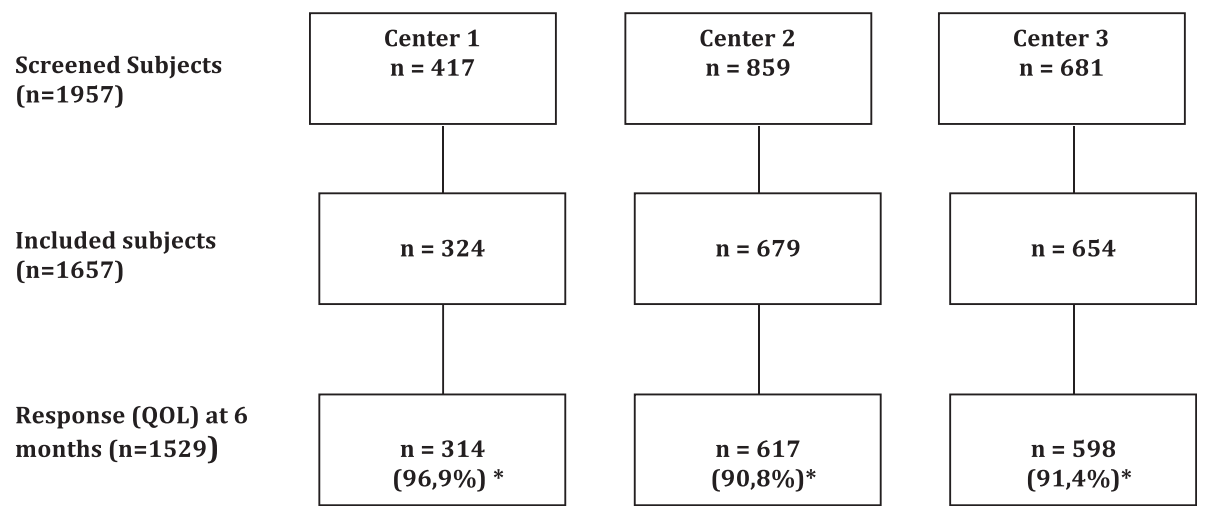

Figure 1 Flowchart of the SATISQOL cohort population. 


\section{Optimism}

Optimism was estimated by the LOT-R (Life Orientation Test Revised) questionnaire. It included 6 statements of personal evaluation concerning general expectations relative to positive or negative consequences. Four statements were included as decoys. Every item scored 0 to 4 . A global score was calculated ranging from 0 (the lowest level of optimism) to 24 (the highest level of optimism). No missing data were allowed for the calculation of this score.

\section{Other data}

Other recorded data included socio-demographic variables, i.e. age, gender, professional activity, family situation, level of education, place of residence, use of psychotropic drugs; and clinical data, i.e. interventions performed during hospital stay, primary chronic disease diagnosis category motivating the hospital stay, and participating center.

\section{Statistical analysis \\ Imputation of missing data}

We calculated the percentage of items not answered to impute the missing data for each dimension of the SF36 and for the STAI score. The percentage of missing data was low, between $0.26 \%$ and $5 \%$. The missing data were considered to be missing completely at random (MCAR), and thus, missing data were imputed with the simple mean of responded items per dimension $[40,41]$.

\section{Descriptive analysis}

First, we selected factors to be adjusted for in the assessment of the relation between HRQOL scores and patient characteristics (socio-demographic and medical data) using a Multiple ANalysis Of VAriance (MANOVA). Variables with a significant relation at an alpha risk of $10 \%$ were retained for multivariate analysis. Associations between HRQOL and both anxiety and optimism were evaluated using linear regression models.

Then, an ANalysis Of VAriance (ANOVA) was performed to examine the relation between HRQOL and the personality traits for each SF36 dimension score. Scores of optimism and anxiety were categorized in 4 classes using median and quartiles. To explain the contribution of these two factors to HRQOL, 3 models were built: firstly, a model assessing the effect of anxiety on HRQOL adjusted for confounders; secondly, a model evaluating the effect of optimism on HRQOL adjusted for confounders; and thirdly, a model assessing the effect of both anxiety and optimism on HRQOL, and their interaction, adjusted for confounders.

All analyses were performed using SAS version 9.3 (SAS Institute Inc., Cary, NC, USA).

\section{Sample size and power of statistical analysis}

Among the 1657 patients included in the SATISQOL cohort, 1529 completed the SF36 questionnaire at 6 months (response rate $=92 \%$ ). This sample size would make it possible to detect a difference of 5 points in HRQOL at an alpha risk of 0.006 (0.05/8, using Bonferroni correction for multiple tests) and a power of $95 \%$ (at a standard deviation of 20 points) to $85 \%$ (at a standard deviation of 25 points).

\section{Results}

\section{Study population}

Of the 1529 patients included in the study, most were aged over 55 years old (61.2\%) and were no longer professionally active (67.5\%). The majority lived at home (98.2\%) and did not live alone (81.2\%). The intervention performed during the index hospitalization was often surgery $(50.5 \%)$. Only $20.4 \%$ had taken psychotropic treatments. Descriptive data are presented in Table 1.

Table 2 shows the distribution of diagnosis category, interventions, and optimism and anxiety scores in each center. In center 3 , the category of diagnosis was mainly rheumatology $(24.1 \%)$ or ear/nose/throat (ENT) and ophthalmology (21.1\%), while in centers 1 and 2, the main diagnosis was cardiovascular $(20.1 \%$ and $38.7 \%$ respectively), endocrinology ( $25.9 \%$ and $16.4 \%$ respectively) or respiratory disease $(24.3 \%$ in center 1$)$. The interventions performed during hospitalisation were: surgery for $78.5 \%$ of patients in center 3 , and for only about $30 \%$ of patients in the other centers. In center 3 , patients were more optimistic, with a score $>17$ observed in $32.3 \%$. They were also less anxious, with a score $>41$ for only $19.4 \%$.

\section{Scores of HRQOL, anxiety and optimism}

The mean HRQOL score was between 48.7 and 66.6 for the 8 dimensions (Table 3 ). The mean anxiety score was 30.1, and the mean optimism score was 15.0.

\section{Relation between HRQOL and personality traits}

There was a significant relation $(\mathrm{p}<0.0001)$ between each dimension of SF36 and the optimism and anxiety scores. HRQOL decreased with increasing levels of anxiety and increased with increasing level of optimism, including for dimensions $\mathrm{MH}$ and $\mathrm{GH}$ (Figure 2).

The variables of adjustment retained for the multivariate models significantly related to HRQOL were: center, age, gender, intervention, level of education, and use of psychotropic drugs. Diagnosis had 10 categories and we did not use this variable in the multivariate model. Likewise, professional activity was also excluded from the multivariate model, as it was significantly related to age and level of education. We did not retain family situation because most of the patients were not living alone.

\section{Model 1 (HRQOL and anxiety)}

The HRQOL score increased for all dimensions of SF36 when the level of anxiety decreased $(\mathrm{p}<0.0001)$ in this model (Tables 4 and 5). The difference in mean HRQOL 
Table 1 Characteristics of the study population

\begin{tabular}{lll}
\hline Variables & Category & $\mathbf{n}(\%)$ \\
\hline Center & 1 & \\
& 2 & $314(20.5)$ \\
& 3 & $617(40.4)$ \\
& & $598(39.1)$ \\
\hline Gender & Men & \\
& Women & $886(58.1)$ \\
& & $639(41.9)$ \\
\hline Age & $<45$ & \\
& $45-55$ & $328(21.5)$ \\
& $55-65$ & $264(17.3)$ \\
& $>65$ & $461(30.1)$ \\
& & $476(31.1)$ \\
\hline
\end{tabular}

Professional activity

Yes $\quad 459(32.5)$

No $953(67.5)$

Family situation

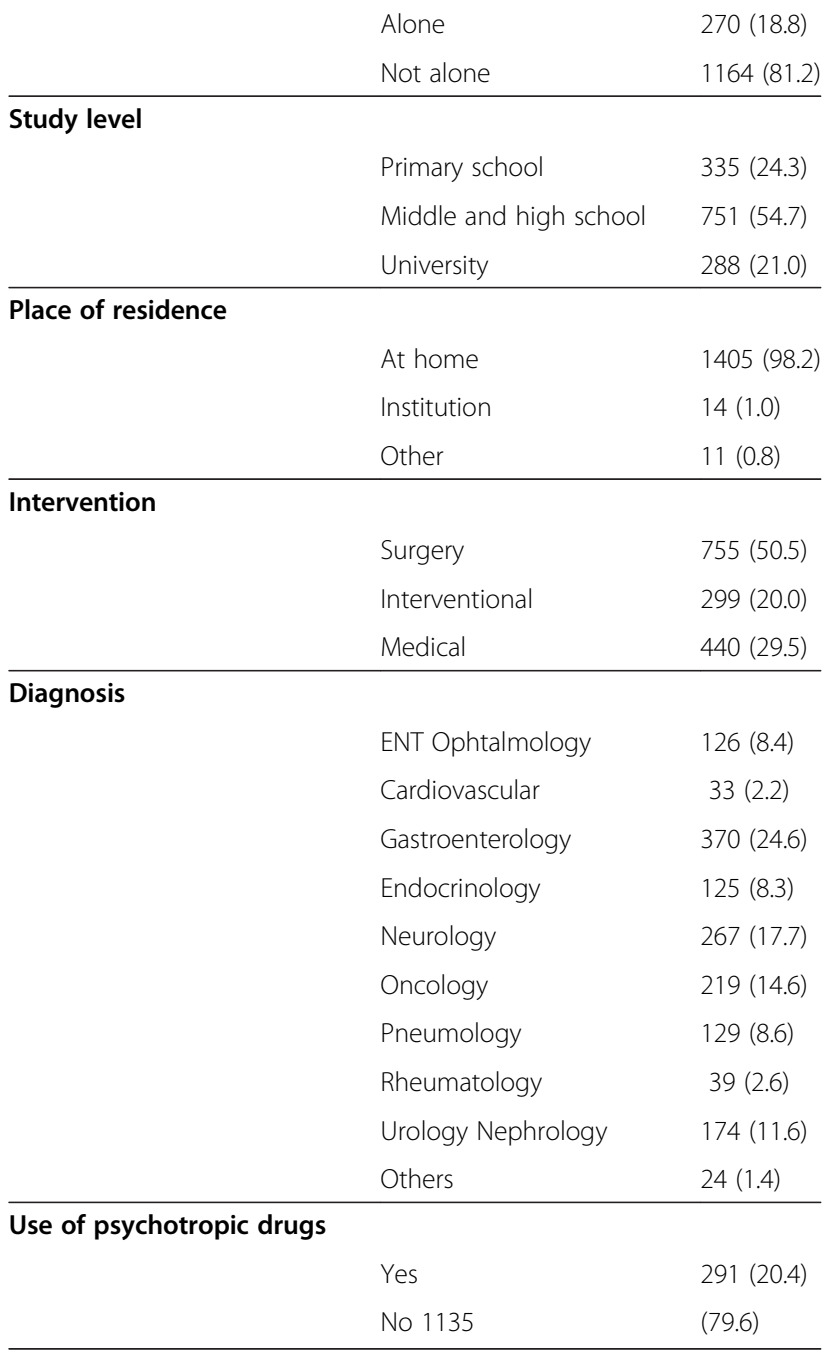

scores between patients with the highest and lowest levels of anxiety was between 16 and 43.5.

\section{Model 2 (HRQOL and optimism)}

The HRQOL score increased for all dimensions of SF36 with increasing level of optimism $(<0.0001)$ (Tables 4 and 5). The difference in mean HRQOL score between patients with the lowest and the highest level of optimism was between 14.6 and 29.8.

\section{Model 3 (HRQOL and anxiety and optimism)}

The HRQOL score for all dimensions of the SF36 increased when the level of anxiety decreased $(\mathrm{p}<0.0001)$ (Tables 4 and 5). The difference in mean HRQOL score between the patients with the lowest and the highest level of anxiety was between 15.8 and 44.5.

The HRQOL score increased with increasing level of optimism $(\mathrm{p}<0.006)$ in the model for all dimensions of SF36 except RP (Tables 4 and 5). The difference in mean HRQOL score between the patients with the lowest and the highest level of optimism was between 3.1 and 12.7.

In this model, interaction between anxiety and optimism was significant for the SF dimension $(p=0.0021)$. For the dimensions $\mathrm{MH}(\mathrm{p}=0.0365)$ and $\mathrm{GH}(\mathrm{p}=0.0300)$, interaction was not significant at the significance level with Bonferroni correction (alpha risk of 0.006). Then, a model for each significant dimension was constructed to study the interaction between optimism and anxiety, which remained significant for the dimensions $\mathrm{GH}\left(\mathrm{p}=0.0049\right.$; $\left.\mathrm{R}^{2} 0.30\right)$, $\mathrm{MH}\left(\mathrm{p}=0.0008 ; \mathrm{R}^{2}=0.53\right)$ and SF $\left(\mathrm{p}=0.0001 ; \mathrm{R}^{2}=0.31\right)$ (Figure 3). For patients with a low level of anxiety, the level of optimism strongly influenced the level of HRQOL for this dimension. A correlation existed between anxiety and optimism $\left(\mathrm{R}^{2}=0.39\right)$.

We also investigated the composite scores. The Physical Composite Score (PCS) increased when the level of anxiety decreased (from 41.7 at the highest level of anxiety to 64.2 at the lowest level of anxiety) ( $p<0.0001)$. Similarly, the PCS increased with the level of optimism (from 63.4 at the lowest level to 71.2 at the highest level of optimism) ( $\mathrm{p}=0.0024)$. The interaction between anxiety and optimism was not significant $(\mathrm{p}=0.4705)$. The Mental Composite Score (MCS) increased when the level of anxiety decreased (from 39.7 at the highest level of anxiety to 67.8 at the lowest level of anxiety) ( $<<0.0001$ ), and increased with the level of optimism (from 52.8 at the lowest level to 59.4 at the highest level of optimism) $(\mathrm{p}<0.0001)$. The interaction between anxiety and optimism was not significant, at the significance level with Bonferroni correction $(\mathrm{p}=0.0476)$.

For this model, as for the other models, HRQOL score was significantly higher for all dimensions except $\mathrm{MH}$ in center 3 than in the other centers $(p<0.03)$, and was significantly better in men for the dimensions PF $(p=0.013)$, 
Table 2 Distribution of diagnosis, intervention, anxiety and optimism scores in each center

\begin{tabular}{|c|c|c|c|c|}
\hline Variables & Category & $\begin{array}{l}\text { Center } 1 \\
\text { n (\%) }\end{array}$ & $\begin{array}{l}\text { Center } 2 \\
\text { n (\%) }\end{array}$ & $\begin{array}{l}\text { Center } 3 \\
\text { n (\%) }\end{array}$ \\
\hline \multicolumn{5}{|l|}{ Diagnosis } \\
\hline & \multicolumn{4}{|l|}{ ENT } \\
\hline & Ophthalomology & $0(0.0)$ & $0(0.0)$ & $126(21.1)$ \\
\hline & Cardiovascular & $62(20.1)$ & $232(38.7)$ & $76(12.7)$ \\
\hline & Gastroenterology & $28(9.1)$ & $178(11.8)$ & $61(10.2)$ \\
\hline & Endocrinology & $80(25.9)$ & $98(16.4)$ & $41(6.9)$ \\
\hline & Neurology & $0(0.0)$ & $2(0,3)$ & $37(6.2)$ \\
\hline & Oncology & $0(0.0)$ & $1(0,2)$ & $32(5.4)$ \\
\hline & Pneumology & $75(24.3)$ & $38(6,3)$ & $12(2.0)$ \\
\hline & Rheumatology & $26(8.4)$ & $3(0,5)$ & $144(24.1)$ \\
\hline & Urology Nephrology & $38(12.3)$ & $45(7.5)$ & $46(7.7)$ \\
\hline & Others & $0(0.0)$ & $2(0.3)$ & $22(3.7)$ \\
\hline \multicolumn{5}{|c|}{ Intervention } \\
\hline & Surgery* & $104(33.7)$ & $182(30.9)$ & $469(78.5)$ \\
\hline & Interventional ** & $32(10.4)$ & $202(34.4)$ & $65(10.9)$ \\
\hline & Medical *** & $173(56.0)$ & $204(34.7)$ & $63(10.5)$ \\
\hline \multicolumn{5}{|c|}{ Anxiety score } \\
\hline \multirow[t]{4}{*}{ (STAI) } & $4-17$ & 75 (23.9) & $157(25.4)$ & $176(29.4)$ \\
\hline & $17-29$ & $84(26.7)$ & $129(20.9)$ & $140(23.4)$ \\
\hline & $29-41$ & $68(21.7)$ & $154(25.0)$ & $166(27.8)$ \\
\hline & $41-80$ & $87(27.7)$ & $177(28.7)$ & $116(19.4)$ \\
\hline \multicolumn{5}{|c|}{ Optimism score } \\
\hline \multirow[t]{4}{*}{ (LOTR) } & $0-13$ & $98(31.2)$ & $175(28.4)$ & $135(22.6)$ \\
\hline & $13-15$ & 49 (15.6) & $117(19.0)$ & 109 (18.2) \\
\hline & $15-17$ & $80(25.5)$ & $180(29.1)$ & $161(26.9)$ \\
\hline & $17-24$ & $87(27.7)$ & 145 (23.5) & $193(32.3)$ \\
\hline
\end{tabular}

* Surgery : patients hospitalized for surgery in connection with the chronic disease.

** Interventional : patients hospitalized for non-surgical intervention (e.g. interventional radiology or non invasive ventilation) in connection with the chronic disease.

*** Medical : patients hospitalized for drug therapy in connection with the chronic disease.

Table 3 Description of dimensions of quality of life and psychological measures

\begin{tabular}{lllll}
\hline & $\mathbf{n}$ & Mean & Standard deviation & Median \\
\hline SF36 score dimensions (0-100) & & & & 28.6 \\
Physical Functioning score & 1486 & 66.6 & 43.2 & 75.0 \\
Role Physical score & 1491 & 48.7 & 27.5 & 50.0 \\
Bodily Pain score & 1511 & 57.0 & 21.6 & 52.0 \\
Vitality score & 1479 & 47.0 & 27.2 & 50.0 \\
Social Functioning score & 1525 & 65.0 & 44.8 & 62,5 \\
Role Emotional score & 1463 & 53.8 & 21.6 & 66,7 \\
Mental Health score & 1470 & 59.7 & 23.4 & 60.0 \\
General Health score & 1429 & 50.6 & 16.2 & 52.0 \\
Anxiety score (4-80) & 1454 & 30.1 & 2.9 & 15.0 \\
Optimism score (0-24) & 1398 & 15.0 & \\
\hline
\end{tabular}




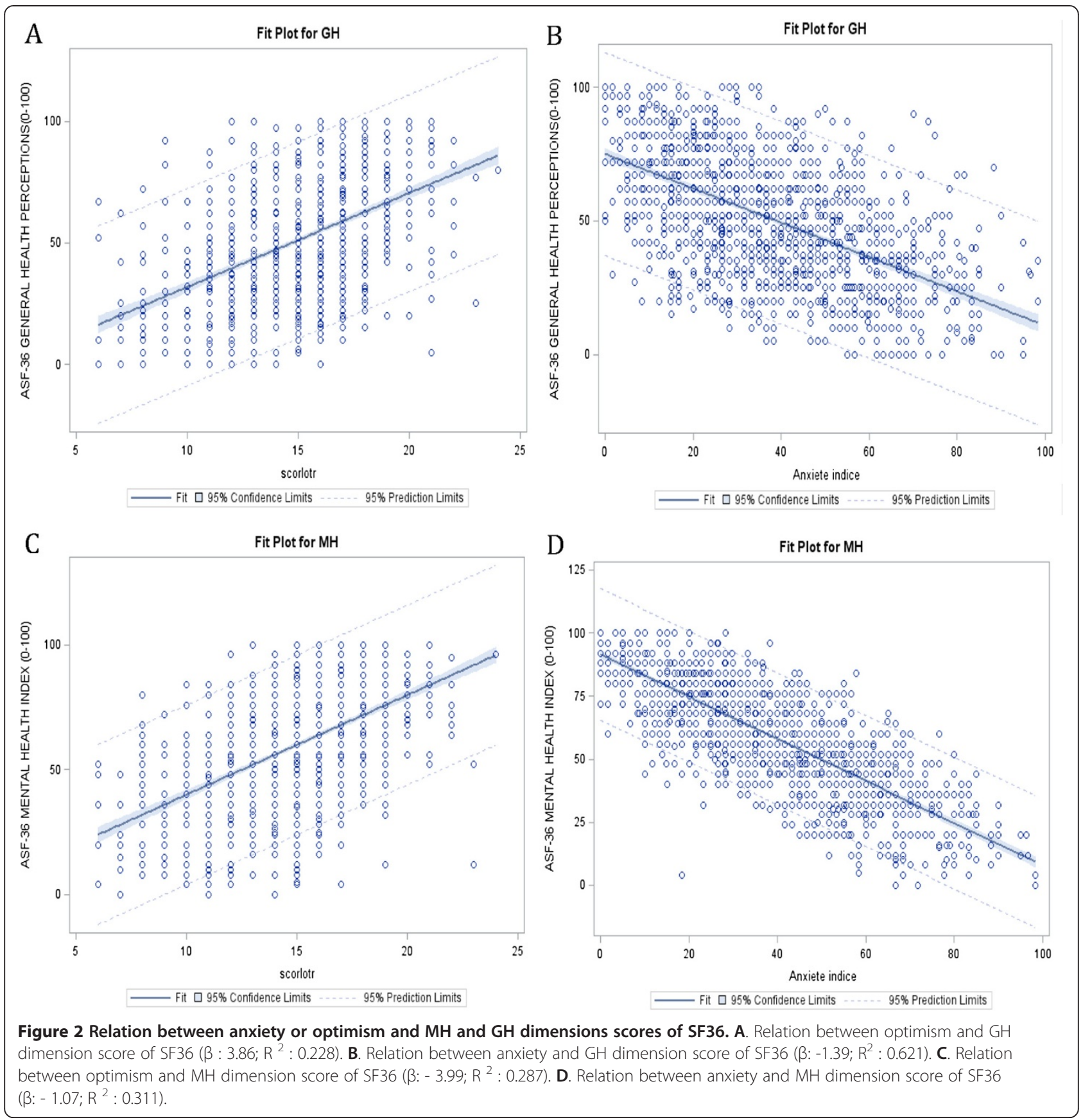

$\mathrm{BP}(\mathrm{p}=0.014), \mathrm{RE}(\mathrm{p}=0.02)$ and $\mathrm{MH}(\mathrm{p}=0.006)$. HRQOL score was significantly higher for younger vs older patients for every dimension except $\mathrm{BP}$ and $\mathrm{MH}(\mathrm{p}<0.05)$. When the intervention performed during hospitalization was surgery, HRQOL was better, except for BP dimension ( $\mathrm{p}<$ 0.05). HRQOL was better among patients with a high level of education for the dimensions PF $(\mathrm{p}=0.0011)$, RP ( $\mathrm{p}=$ $0.0009), B P(p=0.0013)$ and RE $(p=0.0014)$. Patients who were taking psychotropic drugs had a lower level of HRQOL for each dimension $(\mathrm{p}<0.01)$.

\section{Discussion}

\section{Main results of our study}

To the best of our knowledge, this is the first study to specifically examine the relationship between HRQOL and optimism and trait anxiety. By modeling both factors separately and simultaneously, we showed significant associations between personality traits and the HRQOL of patients with chronic disease, especially the effect of anxiety on HRQOL scores, whatever the dimension. These results suggest that for patients hospitalized for chronic disease, a 
Table 4 Association of anxiety or optimism with quality of life (dimensions PF, RP, BP and VT of SF36)

\begin{tabular}{|c|c|c|c|c|c|c|c|c|c|c|c|c|c|}
\hline & & \multicolumn{3}{|c|}{ Physical functioning score } & \multicolumn{3}{|c|}{ Role physical score } & \multicolumn{3}{|c|}{ Bodily pain score } & \multicolumn{3}{|c|}{ Vitality score } \\
\hline & & M1 & M2 & M3 & M1 & M2 & M3 & M1 & M2 & M3 & M1 & M2 & M3 \\
\hline \multicolumn{14}{|l|}{ Anxiety score } \\
\hline & $4-17^{*}$ & 16.0 & & 17.3 & 32.4 & & 35.3 & 22.9 & & 15.8 & 28.6 & & 28.3 \\
\hline & $17-29^{*}$ & 15.1 & & 16.1 & 28.5 & & 29.4 & 19.9 & & 11.4 & 22.1 & & 21.3 \\
\hline & $29-41^{*}$ & 6.8 & & 7.8 & 16.7 & & 17.7 & 8.9 & & -2.7 & 11.8 & & 10.8 \\
\hline & $41-80^{* *}$ & $(54.2)$ & & $(55.2)$ & (26.2) & & $(26.9)$ & $(42.2)$ & & (45.8) & (28.1) & & (29.8) \\
\hline & p-value & $<0,0001$ & & $<0,0001$ & $<0,0001$ & & $<0,0001$ & $<0,0001$ & & $<0,0001$ & $<0,0001$ & & $<0,0001$ \\
\hline \multicolumn{14}{|l|}{ Optimism score } \\
\hline & $0-13^{*}$ & & -14.6 & -3.1 & & -24.9 & -3.8 & & -16.8 & -13.0 & & -19.5 & -5.8 \\
\hline & $13-15^{*}$ & & -6.9 & 2.2 & & -18.5 & -4.5 & & -9.9 & -9.1 & & -14.3 & -0.5 \\
\hline & $15-17^{*}$ & & -4.1 & 0.6 & & -12.7 & -2.5 & & -4.4 & -2.6 & & -7.3 & -0.1 \\
\hline & $17-20^{* *}$ & & (69.2) & (65.6) & & (57.3) & $(50.2)$ & & (61.4) & $(58.1)$ & & (51.9) & $(46.5)$ \\
\hline & $\mathrm{p}$-value & & $<0,0001$ & 0.0038 & & $<0,0001$ & 0.0522 & & $<0,0001$ & 0.0012 & & $<0,0001$ & 0,0001 \\
\hline $\begin{array}{l}\text { Interaction Anxiety score } \\
\text { and Optimism score }\end{array}$ & p-value & & & 0.9872 & & & 0.1792 & & & 0.2807 & & & 0.3709 \\
\hline
\end{tabular}

*Figure in each cells indicates he mean difference with reference class value**.

**reference class : (absolute score value).

M1 (Model 1) : Relation between anxiety and quality of life.

M2 (Model 2) : Relation between optimism and quality of life.

M3 (Model 3) : Relation between anxiety, optimism and quality of life.

All models were adjusted for center, age, gender, level of education, use of psychotropic drugs, p significant $<0.006$.

Dimensions of SF36 score : PF (Physical Functionning), RP (Role Physical), BP (Bodily Pain), VT (Vitality).

Anxiety score: STAI score $<33$ corresponds to the lowest level of anxiety and $>51$ to the highest level of anxiety.

Optimism score : LOT-R score $<13$ corresponds to the lowest level of optimism and $>17$ to the highest level of optimism.

Table 5 Association of anxiety or optimism with quality of life (dimension SF, RE, MH and GH of SF36)

\begin{tabular}{|c|c|c|c|c|c|c|c|c|c|c|c|c|c|}
\hline & & \multicolumn{3}{|c|}{ Social functioning score } & \multicolumn{3}{|c|}{ Role emotional score } & \multicolumn{3}{|c|}{ Mental health score } & \multicolumn{3}{|c|}{ General health score } \\
\hline & & M1 & M2 & M3 & M1 & M2 & M3 & M1 & M2 & M3 & M1 & M2 & M3 \\
\hline \multicolumn{14}{|l|}{ Anxiety score } \\
\hline & $4-33^{*}$ & 34.4 & & 29.2 & 43.5 & & 44.5 & 37.4 & & 37.2 & 26.9 & & 23.8 \\
\hline & $17-29^{*}$ & 30.1 & & 22.1 & 38.9 & & 36.3 & 31.0 & & 28.7 & 20.4 & & 17.1 \\
\hline & $29-41^{*}$ & 17.4 & & 10.7 & 23.4 & & 25.3 & 17.6 & & 15.3 & 10.6 & & 6.2 \\
\hline & $41-80^{* *}$ & $(40.8)$ & & (44.4) & (23.7) & & $(25.2)$ & (36.9) & & $(38.5)$ & $(32.1)$ & & $(35.2)$ \\
\hline & $p$-value & $<0,0001$ & & $<0,0001$ & $<0,0001$ & & $<0,0001$ & $<0,0001$ & & $<0,0001$ & $<0,0001$ & & $<0,0001$ \\
\hline \multicolumn{14}{|l|}{ Optimism score } \\
\hline & $0-13^{*}$ & & -23.0 & -12.7 & & -29.8 & -6.7 & & -24.6 & -6.2 & & -23.4 & -11.8 \\
\hline & $13-15^{*}$ & & -15.0 & -4.7 & & -26.4 & -6.1 & & -16.2 & -1.1 & & -16.4 & -4.7 \\
\hline & $15-17^{*}$ & & -6.7 & -2.6 & & -13.5 & -4.2 & & -9.1 & -1.9 & & -9.9 & -4.0 \\
\hline & $17-20^{* *}$ & & (70.3) & (64.9) & & (64.2) & (55.9) & & $(68.2)$ & (61.1) & & (57.1) & (52.1) \\
\hline & $p$-value & & $<0,0001$ & $<0,0001$ & & $<0,0001$ & 0.0069 & & $<0,0001$ & $<0,0001$ & & $<0,0001$ & $<0,0001$ \\
\hline $\begin{array}{l}\text { Interaction Anxiety score } \\
\text { and Optimism score }\end{array}$ & $p$-value & & & 0.0021 & & & 0.3142 & & & 0.0365 & & & 0.0300 \\
\hline
\end{tabular}

*Figure in each cells indicates he mean difference with reference class value **.

** reference class : (absolute score value).

M1 (Model 1) : Relation between anxiety and quality of life.

M2 (Model 2) : Relation between optimism and quality of life.

M3 (Model 3) : Relation between anxiety, optimism and quality of life.

All models were adjusted for center, age, gender, level of education, use of psychotropic drugs, p significant $<0.006$.

Dimensions of SF36 score : SF, (Social Functionning) RE (Role Emotional), MH (Mental Health), GH (General Health).

Anxiety score: STAI score $<33$ corresponds to the lowest level of anxiety and $>51$ to the highest level of anxiety.

Optimism score : LOT-R score $<13$ corresponds to the lowest level of optimism and $>17$ to the highest level of optimism. 

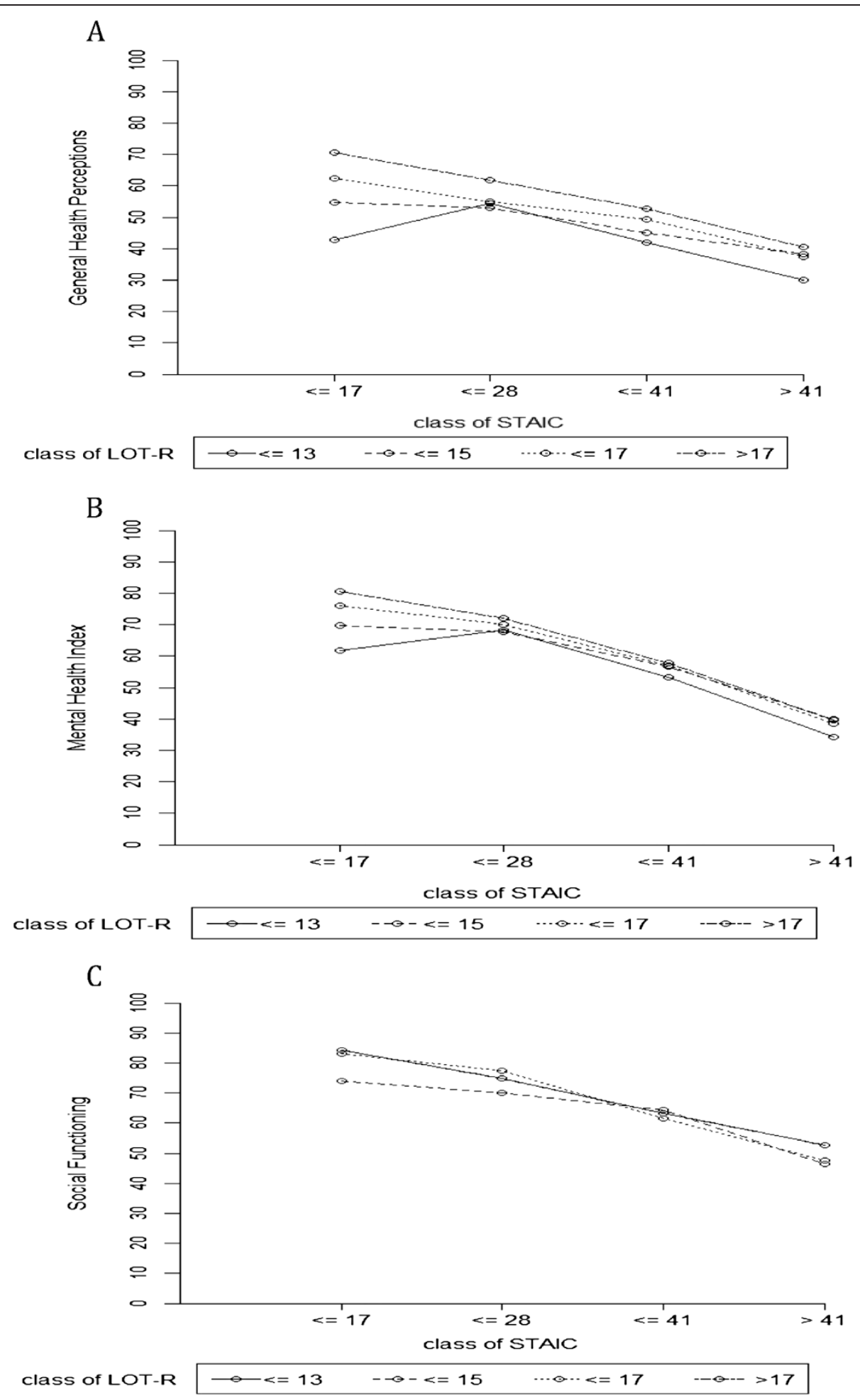

Figure 3 Interaction between optimism and anxiety for the SF, MH, GH dimensions scores of SF36. A. for the GH dimension score of SF36 $\left(p=0.0049 ; R^{2}=0.30\right)$. B. for the MH dimension score of SF36 $\left(p=0.0008 ; R^{2}=0.53\right)$. C. for the SF dimension score of SF36 $\left(p=0.0001 ; R^{2}=0.31\right)$.

high level of anxiety is significantly associated with lower HRQOL for all dimensions. On the other hand, HRQOL increased with increasingly level of optimism, although the relation was less marked than for anxiety. Therefore, anxiety could be a better determinant of HRQOL in this population. 
Furthermore, we took into account the interaction between optimism and anxiety, which was not the case in other published studies. In our study, we found a moderator-interaction effect (optimism) that affects the strength of the relation between a predictor variable (anxiety-trait) and an outcome variable (HRQOL) for some dimensions, in particular for the social dimension, which has never studied before. Thus, at low levels of anxiety, the effect of optimism on HRQOL was amplified. Optimism reduces the negative impact of anxiety on HRQOL.

\section{Data in the literature}

Our results are in agreement with those of previously published studies. First of all, the correlation between the scores of anxiety and optimism was lower in our population than in the validation of the LOTR $\left(R^{2}=0.53\right)$ [13], but higher than in other studies [23,35].

When looking at the HRQOL scores in the general population [39], the scores in our population were lower for anxious patients (between 25 and 55) and more than 50 for less anxious patients (the main score in general population was comprised between 68 and 82 according to the dimension). Similarly, for patients who had a high level of optimism, the HRQOL scores were lower than in the general population, ranging from 50 to 70 .

Furthermore, most studies have focused on the relation between optimism and HRQOL, and have recommended the use of the questionnaire on optimism in the evaluation of HRQOL or to propose adapted care $[4,19,20]$. In this view, pessimism could decrease HRQOL [21,23]. However, although the effect of optimism has often been raised in the evaluation of HRQOL, its impact remains controversial. For women with breast cancer followed-up for 2 years, optimism was not able to predict HRQOL 2 years after the primary operation [26]. Another study reported that after a recent diagnosis of cancer, optimism was correlated with anxiety and HRQOL, but was not a significant predictor at initial diagnosis when other variables like age, functional status, spiritual well-being, depression and anxiety were entered in the model [42].

Finally, fewer studies exist examining the relation between trait anxiety and HRQOL. Most have explored state anxiety, and not trait anxiety as in our study. These studies underlined the effect of anxiety and depression on HRQOL $[3,29,32,34]$, although some reports revealed that interventions like surgery could increase the level of anxiety and depression without decreasing HRQOL. We did not take state anxiety and depression into account in our study. However, we adjusted our analyses for the use of psychotropic drugs, and noted that patients taking psychotropic therapy had a lower HRQOL.

\section{Study limitations}

Our study suffers from several limitations. Previous studies have suggested that HRQOL may be different according to the type of chronic disease $[43,44]$. It can also be estimated differently depending on whether a specific or generic questionnaire is used. For example, in rheumatology, anxiety was more correlated with a specific questionnaire than with the SF36 [45]. Furthermore, we could not take comorbidity into account, and the detriment to HRQOL could be greater in patients with two or more concomitant chronic diseases [6]. Lastly, we were not able to introduce diagnosis into the model, because of the multiplicity of categories. However, we adjusted for the intervention performed during hospitalization, which at least partially reflects the diagnosis.

\section{Impact and strengths of our study}

The strongpoint of our study was to simultaneously study the role of anxiety and optimism in the evaluation of HRQOL.

We had a large multicenter cohort of patients, with a large panel of chronic diseases and thus with high statistical power. A further strongpoint of our study was to evaluate quality of life for many types of disease, using the same generic questionnaire, because most HRQOL studies have investigated a specific disease. Although HRQOL was different between centers participating in the study, we adjusted for this variable, thereby minimizing the potential for bias. Thanks to our use of validated generic questionnaires, our results can be generalized to a large population of patients for many types of chronic disease.

Furthermore, the interaction between trait anxiety and optimism has never yet been studied, even though most studies have reported an effect of optimism on HRQOL. We found that the effect of anxiety on HRQOL was more marked than the effect of optimism. In order to interpret this result appropriately, we must consider trait anxiety as an emotional component, associated with psychological symptoms, whereas optimism is mainly composed by cognitions associated with a perception of the world. Thus, the greater impact of anxiety on quality of life could be due in part to the difference between evaluating an emotional component and a cognitive component.

Although it does not appear essential to measure optimism systematically, it nonetheless seems important to estimate anxiety in the evaluation of HRQOL, because its effect is major. Therefore, we recommend the use in research practice of the trait anxiety questionnaire in future studies evaluating HRQOL, which could be completed by a measure of coping strategies for chronic diseases. The evaluation of coping strategies such as positive reinterpretation, humor associated with optimism or rumination, dramatization associated with more anxiety, would be a 
useful complement to future studies of the impact of personality traits on HRQOL.

\section{Conclusion}

The relation between psychological factors and HRQOL has often been evoked in patients with chronic disease. This is the first study of the relation between HRQOL and the character traits like optimism and anxiety, as well as their interaction, performed in a large cohort of patients with many types of chronic diseases. Optimism and trait anxiety appeared to be significantly correlated with HRQOL. Furthermore, an interaction existed between the trait anxiety and optimism for some dimensions of SF36. Accordingly, HRQOL for less anxious patients was heavily influenced by the level of optimism. Although some studies have revealed the impact of optimism on HRQOL, trait anxiety seems to be a better determinant of HRQOL.

These results could have implications for future studies of HRQOL in patients with chronic diseases. It will be necessary to take account of these results and to evaluate the psychological factors when interpreting HRQOL in large populations of patients followed up for chronic disease.

\begin{abstract}
Abbreviations
HRQOL: Health Related Quality Of Life; SF36: Short form 36; PF: Physical functioning; RP: Role physical; BP: Bodily pain; VT: Vitality; SF: Social functioning; RE: Role emotional; MH: Mental health; GH: General health; STAI: State trait anxiety inventory; LOT-R: Life orientation test revised.
\end{abstract}

\section{Competing interests}

The authors have no potential conflict of interest.

\section{Authors' contributions}

SK participated in the conception and design of the study, acquisition of data, analysis and interpretation of data, drafting of the manuscript and final approval of the manuscript to be published. CB participated in the conception and design of the study, acquisition of data, analysis and interpretation of data, drafting of the manuscript and final approval of the manuscript to be published. AA participated in the analysis and interpretation of data and had given final approval of the manuscript to be published. GB participated in the acquisition of data and final approval of the manuscript to be published. ES participated in the conception and design of the study, revising the manuscript critically for important intellectual content and final approval of the manuscript to be published. PA participated in the conception and design of the study, acquisition of data, revising the manuscript critically for important intellectual content and final approval of the manuscript to be published. FG participated in the conception and design of the study, acquisition of data, revising the manuscript critically for important intellectual content and final approval of the manuscript to be published. MM participated in the conception and design of the study, acquisition of data, analysis and interpretation of data, drafting of the manuscript and final approval of the manuscript to be published. All authors read and approved the final manuscript.

\section{Acknowledgments}

The authors thank all physicians from the centers participating in the SATISQOL study who agreed to invite patients to participate in this study. We thank the clinical research assistants in the 3 centers who participated in the data collection. We also thank Fiona Ecarnot for editorial assistance. This work was supported by the French National Hospital Clinical Research Program.

\section{Author details}

${ }^{1}$ University of Franche-Comté, UPRES EA 3181, Besançon, France. ${ }^{2}$ Emergency and Intensive Care Department CHU, University Hospital Jean Minjoz, 1 Bvd Fleming, Besançon 25030, France. ${ }^{3}$ Université de Lorraine, Université Paris Descartes, EA 4360 APEMAC, Nancy, France. ${ }^{4}$ INSERM, CIC-EC CIE6, Nancy, France. ${ }^{5} \mathrm{CHU}$ de Nancy, Service épidémiologie et évaluation clinique, Nancy, France. ${ }^{6}$ University Hospital Jean Minjoz, Quality of Life and Cancer Plateform, Besançon, France. ${ }^{7}$ Université de Lorraine, Université Paris Descartes, EA 4360 APEMAC-EPSAM, Metz, France. ${ }^{8}$ University of Mediterranee, UPRES EA 3279, Marseille, France.

Received: 12 February 2013 Accepted: 25 July 2013

Published: 5 August 2013

\section{References}

1. Patrick DL, Bergner M: Measurement of health status in the 1990s. Annu Rev Public Health 1990, 11:165-183.

2. Patrick DL, Chiang YP: Measurement of health outcomes in treatment effectiveness evaluations: conceptual and methodological challenges. Med Care 2000, 38(9 Suppl):|114-||25.

3. Benito de Valle M, Rahman M, Lindkvist B, Bjornsson E, Chapman R, Kalaitzakis E: Factors that reduce health-related quality of life in patients with primary sclerosing cholangitis. Clin Gastroenterol Hepatol 2012, 10(7):769-775. e2.

4. Ramirez-Maestre C, Esteve R, Lopez AE: The role of optimism and pessimism in chronic pain patients adjustment. Span J Psychol 2012, 15(1):286-294.

5. Wilsher ML: Psychological stress in sarcoidosis. Curr Opin Pulm Med 2012, 18(5):524-527

6. Rothrock NE, Hays RD, Spritzer K, Yount SE, Riley W, Cella D: Relative to the general US population, chronic diseases are associated with poorer health-related quality of life as measured by the Patient-Reported Outcomes Measurement Information System (PROMIS). J Clin Epidemiol 2010, 63(11):1195-1204.

7. Jerant A, Chapman BP, Franks P: Personality and EQ-5D scores among individuals with chronic conditions. Qual Life Res 2008, 17(9):1195-1204.

8. Bronfenbrenner $U$, et al: Ecological models of human development. International encyclopedia of education 1994, 3:2.

9. McLeroy KR, Bibeau D, Steckler A, Glanz K, et al: An ecological perspective on health promotion programs. Health Educ Q 1988, 15(4):351-377.

10. Wilson IB, Cleary PD, et al: Linking clinical variables with health-related quality of life. A conceptual model of patient outcomes. JAMA 1995, 273(1):59-65.

11. Ferrans CE, Zerwic JJ, Wilbur JE, Larson JL, et al: Conceptual model of health-related quality of life. J Nurs Scholarsh 2005, 37(4):336-342.

12. Carver CS, Smith RG, Antoni MH, Petronis VM, Weiss S, Derhagopian RP: Optimistic personality and psychosocial well-being during treatment predict psychosocial well-being among long-term survivors of breast cancer. Health Psychol 2005, 24(5):508-516.

13. Scheier MF, Carver CS, Bridges MW: Distinguishing optimism from neuroticism (and trait anxiety, self-mastery, and self-esteem): a reevaluation of the life orientation test. J Pers Soc Psychol 1994, 67(6):1063-1078.

14. Spielberger CD: State-trait anger expression inventory : professional manual. Research ed. Odessa Fla (P O Box 998, Odessa 33556): Psychological Assessment Resources; 1988.

15. VanDercar DH, Greaner J, Hibler NS, Spielberger CD, Bloch S: A description and analysis of the operation and validity of the psychological stress evaluator. J Forensic Sci 1980, 25(1):174-188.

16. Maddux RE, Lundh LG, Backstrom M: The swedish depressive personality disorder inventory: psychometrics and clinical correlates from a DSM-IV and proposed DSM-5 perspective. Nord J Psychiatry 2012, 66(3):167-177.

17. Frost MH, Hoskin TL, Hartmann LC, Degnim AC, Johnson JL, Boughey JC: Contralateral prophylactic mastectomy: long-term consistency of satisfaction and adverse effects and the significance of informed decision-making, quality of life, and personality traits. Ann Surg Oncol 2011, 18(11):3110-3116.

18. Beatty $L$, Koczwara B, Rice J, Wade TD: A randomised controlled trial to evaluate the effects of a self-help workbook intervention on distress, coping and quality of life after breast cancer diagnosis. Med J Aust 2010, 193(5 Suppl):S68-S73. 
19. Conversano C, Rotondo A, Lensi E, Della Vista O, Arpone F, Reda MA: Optimism and its impact on mental and physical well-being. Clin Pract Epidemiol Ment Health 2010, 6:25-29.

20. Herzberg PY, Lee SJ, Heussner P, Mumm FH, Hilgendorf I, von Harsdorf S, et al: May personality influences quality-of-life assessments in adult patients after allogeneic hematopoietic SCT: results from a joint evaluation of the prospective German multicenter validation trial and the Fred Hutchinson cancer research center. Bone Marrow Transplant 2013, 48(1):129-134.

21. Vassend O, Quale AJ, Roise O, Schanke AK: Predicting the long-term impact of acquired severe injuries on functional health status: the role of optimism, emotional distress and pain. Spinal Cord 2011, 49(12):1193-1197.

22. Morales Garcia Al, Arenas Jimenez MD, Reig-Ferrer A, Alvarez-Ude F, Malek T, Moledous A, et al: Dispositional optimism in patients on chronic haemodialysis and its possible influence on their clinical course. Nefrologia 2011, 31(2):199-205.

23. Zenger $M$, Glaesmer $H$, Hockel $M$, Hinz A: Pessimism predicts anxiety, depression and quality of life in female cancer patients. J Clin Oncol 2011, 41(1):87-94

24. Schou I, Ekeberg O, Ruland CM: The mediating role of appraisal and coping in the relationship between optimism-pessimism and quality of life. Psychooncology 2005, 14(9):718-727.

25. Jiang $X, H$ e G: Effects of an uncertainty management intervention on uncertainty, anxiety, depression, and quality of life of chronic obstructive pulmonary disease outpatients. Res Nurs Health 2012, 35(4):409-418.

26. Hartl K, Engel J, Herschbach $P$, Reinecker H, Sommer H, Friese K: Personality traits and psychosocial stress: quality of life over 2 years following breast cancer diagnosis and psychological impact factors. Psychooncology 2010, 19(2):160-169.

27. Kostka T, Jachimowicz V: Relationship of quality of life to dispositional optimism, health locus of control and self-efficacy in older subjects living in different environments. Qual Life Res 2010, 19(3):351-361.

28. Horney DJ, Smith HE, McGurk M, Weinman J, Herold J, Altman K, et al: Associations between quality of life, coping styles, optimism, and anxiety and depression in pretreatment patients with head and neck cancer. Head Neck 2011, 33(1):65-71.

29. Mok C, Lok E, Cheung E: Concurrent psychiatric disorders are associated with significantly poorer quality of life in patients with rheumatoid arthritis. Scand J Rheumato/ 2012, 41(4):253-259.

30. Johnson EK, Jones JE, Seidenberg M, Hermann BP: The relative impact of anxiety, depression, and clinical seizure features on health-related quality of life in epilepsy. Epilepsia 2004, 45(5):544-550.

31. Alvarez-Ude F, Fernandez-Reyes MJ, Vazquez A, Mon C, Sanchez R, Rebollo $P$ : Physical symptoms and emotional disorders in patient on a periodic hemodialysis program. Nefrologia 2001, 21(2):191-199.

32. Romao AP, Gorayeb R, Romao GS, Poli-Neto OB, dos Reis FJ, Rosa-e-Silva JC, et al: High levels of anxiety and depression have a negative effect on quality of life of women with chronic pelvic pain. Int J Clin Pract 2009, 63(5):707-711

33. Giardino ND, Curtis JL, Andrei AC, Fan VS, Benditt JO, Lyubkin M, et al: Anxiety is associated with diminished exercise performance and quality of life in severe emphysema: a cross-sectional study. Respir Res 2010, 11:29.

34. Pragodpol P, Ryan C: Critical review of factors predicting health-related quality of life in newly diagnosed coronary artery disease patients. J Cardiovasc Nurs 2013, 28(3):277-284

35. Rajandram RK, Ho SM, Samman N, Chan N, McGrath C, Zwahlen RA: Interaction of hope and optimism with anxiety and depression in a specific group of cancer survivors: a preliminary study. BMC Res Notes 2011, 4:519.

36. Baron RM, Kenny DA, et al: The moderator-mediator variable distinction in social psychological research: conceptual, strategic, and statistical considerations. J Pers Soc Psychol 1986, 51(6):1173-1182.

37. Ware JE Jr, Gandek B: Overview of the SF-36 Health Survey and the International Quality of Life Assessment (IQOLA) Project. J Clin Epidemiol 1998, 51(11):903-912.

38. Wagner AK, Gandek B, Aaronson NK, Acquadro C, Alonso J, Apolone G, et al: Cross-cultural comparisons of the content of SF-36 translations across 10 countries: results from the IQOLA project. International quality of life assessment. J Clin Epidemiol 1998, 51(11):925-932.

39. Leplege A, Ecosse E, Verdier A, Perneger TV: The french SF-36 health survey: translation, cultural adaptation and preliminary psychometric evaluation. J Clin Epidemiol 1998, 51(11):1013-1023.
40. Rubin DB: Inference and missing data. Biometrika 1976, 63:581-592.

41. Little RJ, Rubin DB: Statistical analysis with missing data. New York: John Wiley \& Sons, Inc; 1987

42. Mazanec SR, Daly BJ, Douglas SL, Lipson AR: Relationship between optimism and quality of life in newly diagnosed cancer patients. Cancer Nurs 2010, 33(3):235-243.

43. Norris SL, McNally TK, Zhang X, Burda B, Chan B, Chowdhury FM, et al: Published norms underestimate the health-related quality of life among persons with type 2 diabetes. J Clin Epidemiol 2011, 64(4):358-365.

44. Zingone F, Siniscalchi M, Capone P, Tortora R, Andreozzi P, Capone E, et al: The quality of sleep in patients with coeliac disease. Aliment Pharmacol Ther 2010, 32(8):1031-1036.

45. Nas K, Sarac AJ, Gur A, Cevik R, Altay Z, Erdal A, et al: Psychological status is associated with health related quality of life in patients with rheumatoid arthritis. J Back Musculoskelet Rehabil 2011, 24(2):95-100.

doi:10.1186/1477-7525-11-134

Cite this article as: Kepka et al:: The relationship between traits optimism and anxiety and health-related quality of life in patients hospitalized for chronic diseases: data from the SATISQOL study. Health and Quality of Life Outcomes 2013 11:134.

\section{Submit your next manuscript to BioMed Central and take full advantage of:}

- Convenient online submission

- Thorough peer review

- No space constraints or color figure charges

- Immediate publication on acceptance

- Inclusion in PubMed, CAS, Scopus and Google Scholar

- Research which is freely available for redistribution

Submit your manuscript at www.biomedcentral.com/submit
C) Biomed Central 